\title{
Image Retrieval with Shape Features Extracted using Morphological Operators with BTC
}

\author{
Dr.H.B.Kekre ${ }^{1}$ \\ Sudeep Thepade ${ }^{2}$ \\ ${ }^{1}$ Sr.Professor, ${ }^{2}$ Associate Professor, \\ MPSTME, SVKM'S NMIMS,
}

\section{Mumbai}

\author{
Priyadarshini Mukherjee, \\ Miti Kakaiya, \\ MPSTME, SVKM's NMIMS, \\ Mumbai
}

\author{
Shobhit Wadhwa, \\ Satyajit Singh \\ MPSTME, SVKM's NMIMS, \\ Mumbai
}

\begin{abstract}
The paper discusses novel image retrieval methods based on edge texture of images extracted using morphological operators. The existing CBIR techniques are based on the feature vectors extracted from morphological edge extraction techniques such as simple morphological edge extraction technique, Top-Hat transform and Bottom-Hat transform. The proposed CBIR techniques are using the morphological edge extraction techniques with block truncation coding (BTC). The proposed techniques are tested on generic image database with 1000 images spread across 11 categories. In all 55 queries (5 from each category) are fired on the image database. The average precision and recall of all queries are computed and considered for performance analysis. The experimental results show that use of BTC over morphological shape images for feature extraction improves the performance of image retrieval with reduced computational complexity for query execution. In all BTC with simple morphological edge extraction based CBIR method (SMBTC) gives best performance.
\end{abstract}

\section{Keywords}

CBIR, Top-Hat, Bottom-Hat, BTC, Morphology, Edge extraction

\section{INTRODUCTION}

Information retrieval (IR) is the science of searching for documents, for information within documents, and for metadata about documents, as well as that of searching relational databases and the World Wide Web. There is overlap in the usage of the terms data retrieval, document retrieval, information retrieval, and text retrieval, but each also has its own body of literature, theory and technologies. IR is interdisciplinary, based on computer science, mathematics, cognitive psychology, linguistics, statistics, and physics. Web search engines are the most visible IR applications. Images do have giant share in this information being stored and retrieved, making image retrieval one of the key research field in IR. Content Based image retrieval (CBIR) is the area where searching is done using image content.

The images are very rich in the content like color, texture and shape information present in them [2]. Retrieving images based on color similarity usually involve comparing color histograms [11,16], color averages [4,19], BTC [20] and other methods. Texture measures look for visual patterns in images and how they are spatially defined [14]. The identification of specific textures in an image is achieved primarily by modeling texture as a twodimensional gray level variation, GLCM [10], vector quantization codebooks [6], image transforms [7]. Shape does not refer to the shape of an image but to the shape of a particular region that is being sought out. Shapes are often determined by first applying segmentation or edge detection to an image [12]. Other methods use shape filters to identify given shapes of an image $[13,14]$. In some case accurate shape detection will require human intervention because methods like segmentation are very difficult to completely automate [15]. Here the paper discusses shape texture extraction using morphological operations like erosion, dilation, top hat transform, bottom hat transform. The block truncation coding (BTC) is applied on the extracted shape images to obtain feature vectors of those images which are used for CBIR.

\section{MORPHOLOGICAL IMAGE PROCESSING}

The group of image processing operations which process the image based on shapes is referred as Morphology. In morphological operations the output image is created with help of applying structuring element to input image. In a morphological operation, the value of each pixel in the output image is based on a comparison of the corresponding pixel in the input image with its neighbors. A morphological operation that is sensitive to specific shapes in the input image can be constructed by choosing the size and shape of the neighborhood. Dilation and erosion are the most basic morphological operations. Dilation creates the effect os swelling of shapes of the objects by adding pixels to the boundaries of objects in an image, while erosion forms the object shape shrinking effect by removing pixels on object boundaries. The size and shape of structuring element decide number of pixels added or removed from the objects in an image. In the morphological dilation and erosion operations, the state of any given pixel in the output image is determined by applying a rule to the corresponding pixel and its neighbors in the input image. The rule used to process the pixels defines the operation as dilation or erosion. In dilation the value of the output pixel is the maximum value of all the pixels in the input pixel's neighborhood and in erosion the 
value of the output pixel is the minimum value of all the pixels in the input pixel's neighborhood.

\section{Block Truncation Coding (BTC)}

Block truncation coding (BTC) [1,20] is a simple image coding technique developed in the early years of digital imaging. This method first divides the image to be coded into small non-overlapping image blocks typically of size $4 \times 4$ pixels to achieve reasonable quality. The small blocks are coded one at a time. For each block, the original pixels within the block are coded using a binary bit-map the same Upper Mean Color (UM) size as the original blocks and two mean pixel values. The method first computes the mean pixel value of the whole block and then each pixel in that block is compared to the block mean. If a pixel is greater than or equal to the block mean, the corresponding pixel position of the bitmap will have a value of 1 otherwise it will have a value of 0 . Two mean pixel values one for the pixels greater than or equal to the block mean and the other for the pixels smaller than the block mean are also calculated. At decoding stage, the small blocks are decoded one at a time. For each block, the pixel positions where the corresponding bitmap has a value of 1 is replaced by one mean pixel value and those pixel positions where the corresponding bitmap has a value of 0 is replaced by another mean pixel value. It was quite natural to extend BTC to multi - spectrum images. Most color images are recorded in RGB space, which is perhaps the most wellknown color space. As described previously, BTC divides the image to be coded into small blocks and code them one at a time. For single bitmap BTC of color image, a single binary bitmap the same size as the block is created and two colors are computed to approximate the pixels within the block. To create a binary bitmap in the RGB space, an Inter Band Average Image (IBAI) is first created and a single scalar value is found as the threshold value. The bitmap is then created by comparing the pixels in the IBAI with the threshold value.

\section{EDGE EXTRACTION}

Assume an image $\mathrm{f}$ and a 2-D structuring element $\mathrm{K}$. Erosion followed by dilation represents an important morphological transform called opening, denoted by $f \circ K$. Its dual, closing, denoted by $f \bullet K$, is a dilation followed by an erosion. The residual of the opening compared to the original signal, i.e., $f-(f \circ K)$ represents the top-hat transform. Thus, when the opened signal is subtracted from the original, the desired detail is obtained. Its dual, the bottom-hat transform, is defined as the residual of a closing compared to the original image $f$, i.e., $f-(f \bullet K)$.

Edge extraction is carried out in three ways :

- Dilating the query image and then subtracting the original image from the dilated image.

- $\quad$ Top-hat transform

- Bottom-hat transform

\section{PROPOSED CBIR TECHNIQUE}

The morphological edge extraction based CBIR methods consists of three techniques namely, simple morphological edge extraction (SMEE), Top-Hat Morphological Edge Extraction (THMEE) and Bottom-Hat Morphological Edge Extraction (BHMEE). In the SMEE technique, the original image is subtracted from the dilated image to give us the feature vector. In THMEE, Top-Hat transform is applied onto the image. In BHMEE, Bot-Hat transform is applied onto the image. These existing techniques can be extended using BTC[1]. Hence the novel proposed techniques are as follows:

$$
\begin{array}{ll}
- & \text { SMEE with BTC (SM BTC) } \\
- & \text { THMEE with BTC (TH BTC) } \\
\text { - } & \text { BHMEE with BTC (BH BTC) }
\end{array}
$$

Table 1 shows the feature vector sizes of both existing and the proposed CBIR techniques. The feature vector size in the existing morphological edge extraction based CBIR techniques is $256 \times 256$. Thus in general, for an $\mathrm{NxN}$ image the feature vector size is $\mathrm{N}^{2}$. The feature vector size of the proposed techniques is reduced to 6 which in turn reduces the time required for query execution. Also the feature vector size is independent of the size the image.

Table 1: Feature Vector size for proposed CBIR
techniques
\begin{tabular}{|c|c|c|}
\hline $\begin{array}{c}\text { CBIR } \\
\text { Technique } \\
\text { Using }\end{array}$ & $\begin{array}{c}\text { Morphological } \\
\text { Operators } \\
\text { [SMEE / THMEE } \\
\text { / BHMEE] }\end{array}$ & $\begin{array}{c}\text { BTC with } \\
\text { Morphological } \\
\text { Operators } \\
\text { [SMBTC / THBTC } \\
\text { / BHBTC] }\end{array}$ \\
\hline $\begin{array}{c}\text { Feature Vector } \\
\text { size for NxN } \\
\text { image }\end{array}$ & $\mathrm{N}^{2}$ & 6 \\
\hline
\end{tabular}

\section{IMPLEMENTATION}

The discussed image retrieval methods are implemented using MATLAB 7.0 on Intel Core 2 Duo processor T8100(2.1 GHz) with $2 \mathrm{~GB}$ of RAM. To check the performance of proposed technique a database of 1000 variable sized images spread across 11 categories has been used [3]. Five queries were selected from each category of images. Mean Squared Error (MSE) is used as similarity measure for comparing the query image with all the images in the image database. Let Vpi and Vqi be the feature vectors of image ' $\mathrm{P}$ ' and Query image ' $\mathrm{Q}$ ' respectively with size ' $n$ ', then the MSE can be given as shown in equation 1 .

$$
M S E=\sum_{i=1}^{n}(V p i-V q i)^{2}
$$

To assess the retrieval effectiveness, we have used the precision and recall as statistical comparison parameters for our proposed technique of CBIR. The standard definitions of these two measures are given by following equations. 
Precision $=\frac{\text { Number of relevant images retrieved }}{\text { Total number of } \text { images retrieved }}$

Recall $=\frac{\text { Number of relevant images retrieved }}{\text { Total number of relevant images in database }}$

\section{RESULTS AND DISCUSSIONS}

Figure 1 is a graph plotted between average precision and recall and the number of images retrieved. It shows the comparison between Simple Morphological Edge Extraction (SMEE) and Simple Morphological Edge Extraction with BTC (SM BTC). The crossover point between precision and recall for SM BTC is higher then SMEE. This indicates a performance enhancement in case of the proposed techniques over the existing techniques.

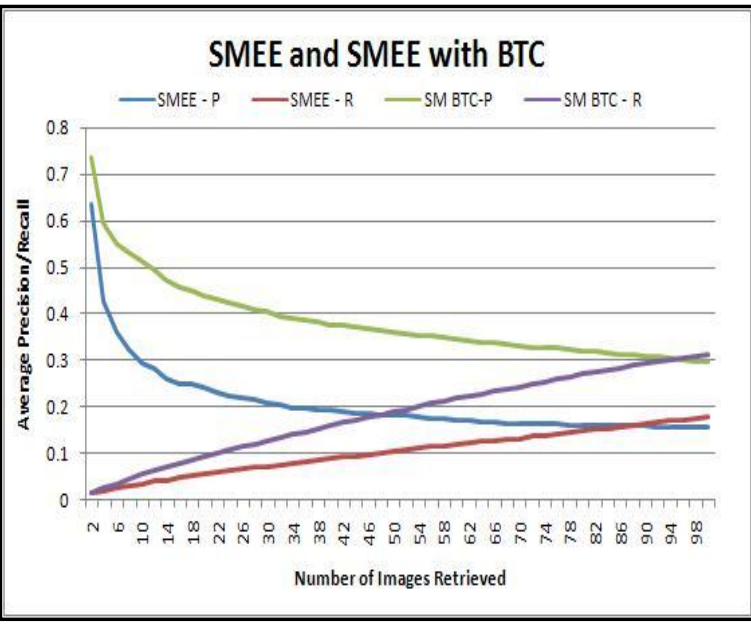

Figure 1: Comparison between simple edge extraction and BTC edge extraction

The crossover points for Bottom-Hat Morphological Edge Extraction with BTC (BH BTC) is higher than the crossover point for Bottom-Hat Morphological Edge Extraction as shown in figure 2 which shows a graph plotted between precision and recall and number of images retrieved. This figure also indicates a performance enhancement in case of the proposed techniques over the existing techniques.

As shown in figure 3, which is a graph plotter between average precision and recall and number of images retrieved for Top-Hat morphological edge extraction with BTC (TH BTC) and Top-Hat morphological Edge Extraction (THMEE), TH BTC gives a better crossover point that THMEE. Here too it is indicated that the performance of the proposed techniques is better than the existing techniques.

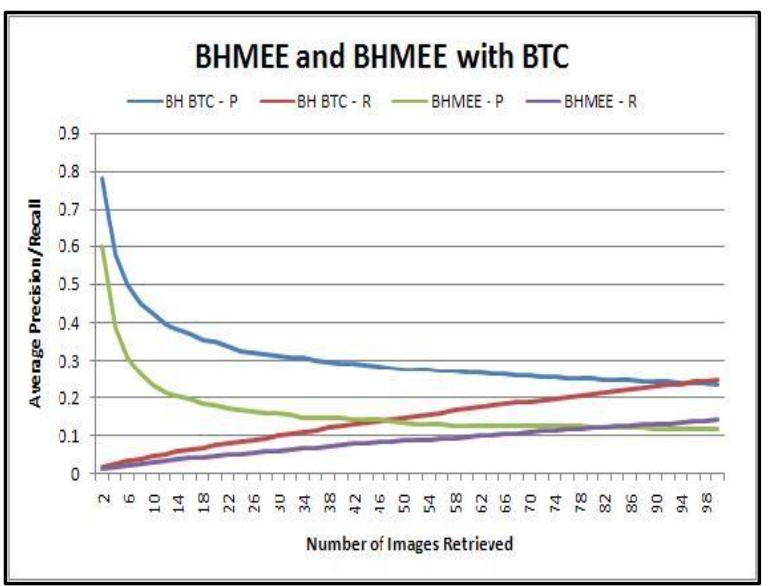

Figure 2: Comparison between simple Bottom-hat and BTC Bottom-hat

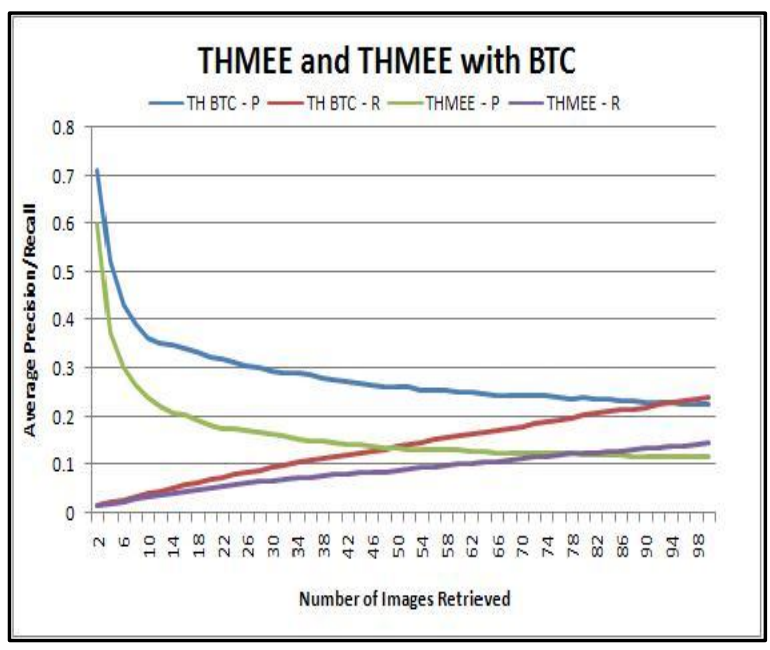

Figure 3: Comparison between simple Top-hat and BTC Top-hat

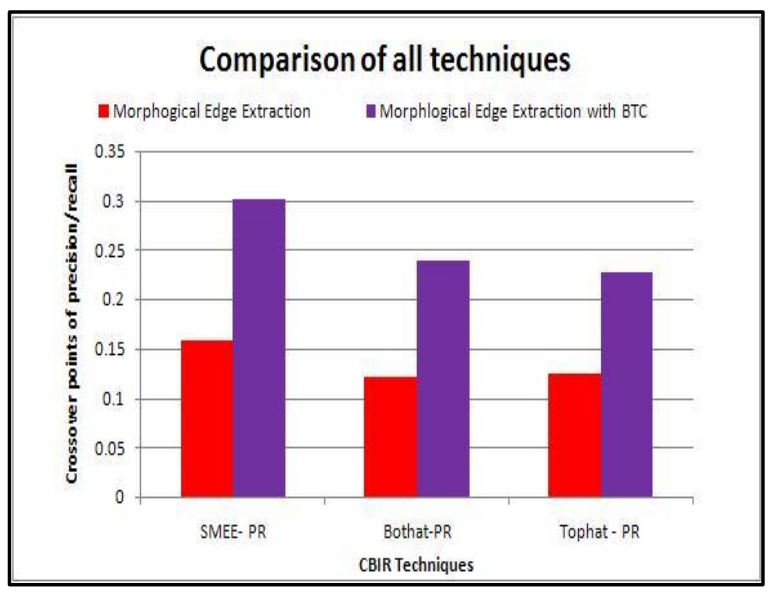

Figure 4: Comparison of simple morphological techniques with BTC 
The crossover points are of utmost importance as they help us determine the performance of each of the CBIR techniques. Hence figure 4 shows a bar chart which shows the comparison between the crossover points of the existing and the proposed CBIR techniques. As is evident from the figure, Morphological Edge Extraction with BTC gives a much better performance than the simple Morphological Edge Extraction techniques.

\section{CONCLUSION}

The improvement in the performance of image retrieval and the speed of image retrieval are two main thirst areas for researchers working on CBIR. Generally achieving better image retrieval results gainsay the faster image retrieval. The paper shows how the performance of image retrieval can be improved even with reduction in retrieval time. Here block truncation coding is used over the morphological shape images for feature extraction resulting in smaller feature vectors and better performance of image retrieval indicated by higher precision and recall values.

\section{REFERENCES}

[1]. Dr.H.B.Kekre, Sudeep D. Thepade, Priyadarshini Mukherjee, Shobhit Wadhwa, Miti Kakaiya, Satyajit Singh, "Image Retrieval with Shape Features Extracted using Gradient Operators and Slope Magnitude Technique with BTC", International Journal of Computer Applications, September 2010 issue.

[2]. Dr.H.B.Kekre, Sudeep D. Thepade, "Rendering Futuristic Image Retrieval System", National Conference on Enhancements in Computer, Communication and Information Technology, EC2IT-2009, 20-21 Mar 2009, K.J.Somaiya College of Engineering, Vidyavihar, Mumbai-77.

[3]. Image database http://wang.ist.psu.edu/docs/related/Image.orig (Last referred on 23 Sept 2008)

[4]. Dr.H.B.Kekre, Sudeep D. Thepade, Archana Athawale, Anant Shah, Prathmesh Verlekar, Suraj Shirke,"Energy Compaction and Image Splitting for Image Retrieval using Kekre Transform over Row and Column Feature Vectors", International Journal of Computer Science and Network Security (IJCSNS), Volume:10, Number 1, January 2010, (ISSN: 1738-7906) Available at www.IJCSNS.org.

[5]. Dr.H.B.Kekre, Sudeep D. Thepade, "Image Retrieval using Color-Texture Features Extracted from Walshlet Pyramid", ICGST International Journal on Graphics, Vision and Image Processing (GVIP), Volume 10, Issue I, Feb.2010, pp.9-18, Available

online www.icgst.com/gvip/Volume10/Issue1/P11509388 76.html

[6]. Dr.H.B.Kekre, Tanuja Sarode, Sudeep D. Thepade, "Color-Texture Feature based Image Retrieval using DCT applied on Kekre's Median Codebook", International Journal on Imaging (IJI), Volume 2,
Number A09, Autumn 2009,pp. 55-65. Available online at www.ceser.res.in/iji.html

[7]. Dr.H.B.Kekre, Sudeep D. Thepade, "Image Retrieval using Non-Involutional Orthogonal Kekre's Transform", International Journal of Multidisciplinary Research and Advances in Engineering (IJMRAE), Ascent Publication House, 2009, Volume 1, No.I, pp 189-203, 2009. Abstract available online at www.ascent-journals.com

[8]. Dr.H.B.Kekre, Sudeep D. Thepade, "Improving the Performance of Image Retrieval using Partial Coefficients of Transformed Image", International Journal of Information Retrieval, Serials Publications, Volume 2, Issue 1, 2009, pp. $72-79$

[9]. Dr.H.B.Kekre, Sudeep D. Thepade, Archana Athawale, Anant Shah, Prathmesh Verlekar, Suraj Shirke, "Performance Evaluation of Image Retrieval using Energy Compaction and Image Tiling over DCT Row Mean and DCT Column Mean", Springer-International Conference on Contours of Computing Technology (Thinkquest2010), Babasaheb Gawde Institute of Technology, Mumbai, 13-14 March 2010, The paper will be uploaded on online Springerlink.

[10]. Dr.H.B.Kekre, Tanuja K. Sarode, Sudeep D. Thepade, Vaishali Suryavanshi,"Improved Texture Feature Based Image Retrieval using Kekre's Fast Codebook Generation Algorithm", SpringerInternational Conference on Contours of Computing Technology (Thinkquest-2010), Babasaheb Gawde Institute of Technology, Mumbai, 13-14 March 2010, The paper will be uploaded on online Springerlink.

[11]. Hirata K. and Kato T. "Query by visual example content-based image retrieval", In Proc. Of Third International Conference on Extending Database Technology, EDBT'92, 1992, pp 56-71.

[12]. Sagarmay Deb, Yanchun Zhang, "An Overview of Content Based Image Retrieval Techniques," Technical Report, University of Southern Queensland.

[13]. Rafael C. Gonzalez, Richard E. Woods, "Digital Image Processing". Chapter 10, pg 599-607. Published by Pearson Education, Inc. 2005.

[14]. William I. Grosky, "Image Retrieval - Existing Techniques, Content-Based (CBIR) Systems" Department of Computer and Information Science, University of Michigan-Dearborn, Dearborn, MI, USA,http://encyclopedia.jrank.org/articles/pages/67 63/Image-Retrieval.html\#ixzz0130drFVs, referred on 9 March 2010

[15]. Bill Green, "Canny Edge Detection Tutorial”, 2002. http://www.pages.drexel.edu/ weg22/can_tut.html, referred on 9 March 2010

[16]. John Eakins, Margaret Graham, "Content Based Image Retrieval", Chatpter 5.6, pg 36-40, University of Northrumbia at New Castle, October 1999 
[17]. Dr.H.B.Kekre, Sudeep D. Thepade, Akshay Maloo, "Performance Comparison of Image Retrieval Techniques using Wavelet Pyramids of Walsh, Haar and Kekre Transforms", International Journal of Computer Applications (IJCA) Volume 4, Number 10, August 2010 Edition, pp 1-8, http://www.ijcaonline.org/archives/volume4/numbe r10/866-1216

[18]. Dr.H.B.Kekre, Sudeep D. Thepade, Akshay Maloo, "Performance Comparison of Image Retrieval Using Fractional Coefficients of Transformed Image Using DCT, Walsh, Haar and Kekre's Transform", CSC International Journal of Image Processing (IJIP), Volume 4, Issue 2, pp 142-157, Computer Science Journals, CSC Press, www.cscjournals.org

[19]. Dr.H.B.Kekre, Sudeep D. Thepade, Varun K. Banura, "Amelioration of Colour Averaging Based Image Retrieval Techniques using Even and Odd parts of Images", International Journal of Engineering Science and Technology (IJEST), Vol. 2, Issue 9, Sept. 2010. pp. (ISSN: 0975-5462) Available online at http://www.ijest.info.

[20]. Dr.H.B.Kekre, Sudeep D. Thepade, "Boosting Block Truncation Coding using Kekre's LUV Color Space for Image Retrieval", WASET International Journal of Electrical, Computer and System Engineering (IJECSE), Vol. 2, No.3, Summer 2008. Available online at www.waset.org/ijecse/v2/v2-323.pdf

\section{Author Biographies}

Dr. H. B. Kekre has received B.E. (Hons.) in Telecomm. Engineering. from Jabalpur University in 1958, M.Tech (Industrial Electronics) from IIT Bombay in 1960, M.S.Engg. (Electrical Engg.) from University of Ottawa in 1965 and Ph.D. (System Identification) from IIT Bombay in $1970 \mathrm{He}$ has worked as Faculty of Electrical Engg. and then HOD Computer Science and Engg. at IIT Bombay. For 13 years he was working as a professor and head in the Department of Computer Engg. at Thadomal Shahani Engineering. College, Mumbai. Now he is Senior Professor at MPSTME, SVKM's NMIMS University. He has guided 17 Ph.Ds, more than 100 M.E./M.Tech and several B.E./B.Tech projects. His areas of interest are Digital Signal processing, Image Processing and Computer Networking. He has more than 320 papers in National / International Conferences and Journals to his credit. He was Senior Member of IEEE. Presently He is Fellow of IETE and Life Member of ISTE Recently ten students working under his guidance have received best paper awards and two have been conferred Ph.D. degree of SVKM's NMIMS University. Currently 10 research scholars are pursuing Ph.D. program under his guidance.
Sudeep D. Thepade has Received B.E.(Computer) degree from North Maharashtra University with Distinction in 2003. M.E. in Computer Engineering from University of Mumbai in 2008 with Distinction, currently pursuing Ph.D. from SVKM's NMIMS, Mumbai. He has about than 07 years of experience in teaching and industry. He was Lecturer in Dept. of Information Technology at Thadomal Shahani Engineering College, Bandra(w), Mumbai for nearly 04 years. Currently working as Associate Professor in Computer Engineering at Mukesh Patel School of Technology Management and Engineering, SVKM's NMIMS University, Vile Parle(w), Mumbai, INDIA. He is member of International Association of Engineers (IAENG) and International Association of Computer Science and Information Technology (IACSIT), Singapore. He is reviewer for many international journals and in the international advisory panel for many international conferences. His areas of interest are Image Processing and Computer Networks. He has about 90 papers in National/International Conferences/Journals to his credit with a Best Paper Award at Int. Conference SSPCCIN-2008, Second Best Paper Award at ThinkQuest-2009 National Level faculty paper presentation competition and Best Paper Award at Springer Int. Conf. ICCCT-2010.

Shobhit Wadhwa is pursuing a B.Tech degree in Information Technology from MPSTME, SVKM's NMIMS University, Mumbai, India. His areas of interest lie in image processing and information systems development. He is also a member of the IEEE committee of his college.

Satyajit Singh is pursuing a B.Tech degree in Information Technology from MPSTME, SVKM's NMIMS University, Mumbai,India. His areas of interest lie in the fields of Image processing and Wireless technologies.

Priyadarshini Mukherjee is pursuing a B.Tech degree in Information Technology from MPSTME, SVKM's NMIMS University, Mumbai. Her interests lie in the fields of image processing and website development.

Miti Kakaiya is pursuing a B.Tech degree in Information Technology from MPSTME, SVKM's NMIMS University, Mumbai. Her interests lie in the field of Image processing and website designing. 